\title{
First atmospheric observations of three chlorofluorocarbons
}

\author{
J. C. Laube and A. Engel \\ Institute for Atmosphere and Environment, University of Frankfurt (Main), Germany \\ Received: 26 February 2008 - Published in Atmos. Chem. Phys. Discuss.: 7 April 2008 \\ Revised: 1 July 2008 - Accepted: 7 August 2008 - Published: 2 September 2008
}

\begin{abstract}
We report the first atmospheric observations of the Chlorofluorocarbons (CFCs) trifluorochloroethene, 3-chloropentafluoropropene and 4,4-dichlorohexafluoro-1butene by means of Gas Chromatography with Electron Capture and Mass Spectrometric detection (GC-ECD-MS) in air samples taken at the Taunus Observatory operated by the University of Frankfurt (Main) and the Jungfraujoch High Altitude Research Station in Switzerland. These substances belong to a class of CFCs containing a double bond and are suspected to originate from the production and thermal degradation of widely used fluoropolymers like polychlorotrifluoroethene (PCTFE). Their atmospheric lifetimes are expected to be rather short. A quantitative calibration could only be derived for trifluorochloroethene but not for the other species by now. Thus, we use a relative sensitivity method to get a first indication of the observed atmospheric abundances. Identification was possible because of an air plume containing high concentrations of these substances. We suggest that the abundances found on this occasion originated from a local source. However, we have also observed the novel CFCs in air masses representative of background conditions, though with much lower concentrations. These species and some of their degradation products are toxic and could also be relevant for stratospheric and tropospheric ozone depletion.
\end{abstract}

\section{Introduction}

The production and use of many halocarbons is regulated by the Montreal Protocol on Substances that Deplete the Ozone Layer and its subsequent amendments (see http:// ozone.unep.org/ for details), because if these substances are transported into the stratosphere, they can release chlorine and/or bromine. The inorganic chlorine/bromine species released from the halocarbons enhance the efficiency of catalytic ozone destruction cycles. This has led to both global ozone depletion and strong loss of nearly all ozone in an altitude layer between 15 and $25 \mathrm{~km}$ above Antarctica during austral spring; a phenomenon known as the ozone hole. See e.g. Solomon, 1999 for a detailed review on this subject. Up to date only seven Chlorofluorocarbons (CFCs) have been observed in the atmosphere (e.g. Clerbaux and Cunnold, 2007). These CFCs have rather long atmospheric lifetimes of more than 40 years. Five of them $-\mathrm{CF}_{2} \mathrm{Cl}_{2}$ (CFC12), $\mathrm{CFCl}_{3}$ (CFC-11), $\mathrm{CF}_{2} \mathrm{ClCF}_{2} \mathrm{Cl}$ (CFC-114), $\mathrm{CF}_{3} \mathrm{CFCl}_{2}$ (CFC-114a) and $\mathrm{CF}_{2} \mathrm{ClCFCl}_{2}$ (CFC-113) - are decreasing in the global background atmosphere due to their regulation under the Montreal Protocol on Substances that Deplete the Ozone Layer (see Table 1.2 of Clerbaux and Cunnold, 2007 for details). The only exception is $\mathrm{C}_{2} \mathrm{~F}_{5} \mathrm{Cl}$ (F115) which was recently reported to have stabilised in concentration (Reimann et al., 2007). This is due to its very long atmospheric lifetime. For $\mathrm{CF}_{3} \mathrm{Cl}(\mathrm{CFC}-13)$ there is no upto-date trend data available. Here we report the first atmospheric observations of three CFCs which contain a double bond and are thus expected to have short atmospheric lifetimes relative to atmospheric transport times (i.e. below half a year). Considerable amounts of such short-lived substances are able to reach the stratosphere. Law and Sturges, 2007 estimated about 55 parts per trillion (ppt; range: $52-60 \mathrm{ppt}$ ) of chlorine from 10 short-lived substances to be present in the main stratospheric entrance region, the tropical upper troposphere. Thus, although their ozone depletion potential might be small, the newly observed short-lived CFCs are potential contributors to stratospheric chlorine. 


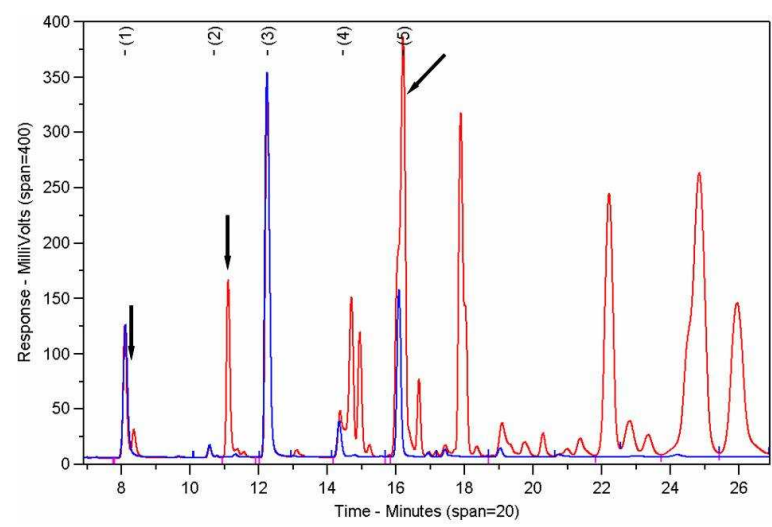

Fig. 1. Comparison of the chromatograms from two air samples taken at the Taunus Observatory operated by the University of Frankfurt. Trace gases were concentrated cryogenically from one litre of air and detected using an ECD. The blue line represents unpolluted air while the red line is the plume sample taken on the 02 October 2007 which showed a number of unidentified large signals. The known large peaks are $\mathrm{CF}_{2} \mathrm{Cl}_{2}$ [CFC-12, (1)], $\mathrm{CF}_{2} \mathrm{ClBr} / \mathrm{CF}_{2} \mathrm{ClCF}_{2} \mathrm{Cl}$ [Halon-1211/CFC-114, coeluting, (2)], $\mathrm{CFCl}_{3}$ [CFC-11, (3)], $\mathrm{CF}_{2} \mathrm{ClCFCl}_{2}$ [CFC-113, (4)] and $\mathrm{CCl}_{4}$ (5). Three of the unknown substances could be identified by now and are labelled with black arrows: trifluorochloroethene at $8.4 \mathrm{~min}, 3-$ chloropentafluoropropene at $11.1 \mathrm{~min}$ and 4,4-dichlorohexafluoro1-butene at $16.2 \mathrm{~min}$.

\section{Analytical procedure}

Air samples were taken at the Taunus Observatory at the Kleiner Feldberg (50 $\left.13^{\prime} 29.6^{\prime \prime} \mathrm{N}, 8^{\circ} 26^{\prime} 28.7^{\prime \prime} \mathrm{E}\right)$ near Frankfurt (Main), Germany between October and December 2007. Stainless steel and also silanized stainless steel canisters were used for sampling. The canisters were tested prior to use and none of them showed a blank signal in any CFC. The canisters were evacuated using a turbo pump which was also checked to be free of CFCs. They were filled by simply opening them in order to avoid effects from a compressor or a drying agent. A CFC-free and preconditioned stainless steel sampling line was used to avoid breath contaminations. Samples were not dried before measuring and no pressure regulator was used. The calibration standard was measured with and without attached pressure regulator. No significant concentration difference in any CFC was observed proving that the pressure regulator did not gas out these substances. For analysis trace gases out of $500-1000 \mathrm{ml}$ of air were pre-concentrated cryogenically on porous glass beads (mesh size: 60$)$ inside a sample loop (1/8" stainless steel, length: $\sim 30 \mathrm{~cm}$ ) by cooling it with liquid nitrogen. The pre-concentration system consisted almost completely of stainless steel parts and was evacuated to about $4 \times 10^{-2}$ mbar prior to analysis using a Leybold Trivac pump, type D4B with an activated alumina sorbent filter to prevent back streaming. Due to the low pressure $\mathrm{N}_{2}, \mathrm{O}_{2}, \mathrm{H}_{2}$ and three noble gases ( $\mathrm{He}, \mathrm{Ne}$ and $\mathrm{Ar}$ ) remained in gaseous state and passed the sample loop while the higher boiling trace gases were condensed. The uncondensed compounds reached a reference volume canister with a pressure sensor (range: 0 to 1.7 bar, full scale accuracy: $0.073 \%$, model 204 from SETRA Systems Inc., USA). As these compounds represent more than 99.9 Vol.- $\%$ of dry air the pressure inside the reference volume can be used as a direct measure of the air volume that passed the sample loop. Separation was carried out with a Siemens Sil Gas Chromatograph on a $1 / 8^{\prime \prime}$ micro packed Porasil C/n-Octane column (length: $5 \mathrm{~m}$ ) with a temperature programme from $-40^{\circ} \mathrm{C}$ to $120^{\circ} \mathrm{C}$. The carrier gas was Helium (ALPHAGAZ 2 from Air Liquide Deutschland $\mathrm{GmbH}$ ) with a purity $\geq 99.9999 \%$-mol which was further purified from oxygen, hydrocarbons and moisture using a gas purification system from Chromatography Research Supplies, USA. Two parallel detectors were used simultaneously: an Electron Capture detector (ECD) from Siemens and a quadrupole Mass Spectrometer (MS) from Agilent (5975 inert XL mass selective detector with Performance turbo pump and High vacuum gauge controller from Agilent Technologies) run in electron impact selected ion monitoring (EI-SIM) mode. The carrier gas flow was regulated to about $6 \mathrm{ml} / \mathrm{min}$ with a detector split of about $2.5 / 3.5 \mathrm{ml} / \mathrm{min}$ (MS/ECD). This ratio was achieved by varying the length of the transfer capillaries from the splitter to the detectors (inner diameter of $0.15 \mathrm{~mm}$, length to ECD: $\sim 70 \mathrm{~cm}$, length to MS: $\sim 100 \mathrm{~cm}$ ). The flow rates vary slightly with temperature as the system contains no electronic pressure control (EPC) valves. The ECD detector was additional flushed with ultra-pure Nitrogen $(30 \mathrm{ml} / \mathrm{min}$, from Air Liquide Deutschland $\mathrm{GmbH}$, purity $\geq 99.999 \%$-mol which was further purified similar to Helium) as a so-called "make-up" flow. The chromatographic system separates substances primary via boiling points and covers a range from -90 to $150^{\circ} \mathrm{C}$. MS detection limits were below $1 \mathrm{ppt}$ for almost every halocarbon and below $0.3 \mathrm{ppt}$ for the six measurable CFCs (except CFC-13; ECD detection limits of the six CFCs were below $0.1 \mathrm{ppt}$ ). Different blanks were carried out by injecting vacuum or pre-concentrating carrier and make-up gas but none of the detectors showed a blank signal in any CFC.

\section{Results and discussion}

On the 2 October 2007 an air sample was taken at the Taunus Observatory. During analysis using the system described above the ECD showed a number of additional signals with a high abundance. Figure 1 shows the zoom of the ECD chromatogram in comparison with a unpolluted air sample. Several large signals occurred in addition to the known peaks belonging to $\mathrm{CF}_{2} \mathrm{Cl}_{2}$ [CFC-12, (1)], $\mathrm{CF}_{2} \mathrm{ClBr} / \mathrm{CF}_{2} \mathrm{ClCF}_{2} \mathrm{Cl}$ [Halon-1211/CFC-114, coeluting, (2)], $\mathrm{CFCl}_{3}$ [CFC-11, (3)], $\mathrm{CF}_{2} \mathrm{ClCFCl}_{2}$ [CFC-113, (4)] and $\mathrm{CCl}_{4}(5)$. This was a strong indication for the presence 
halocarbons as the ECD is very sensitive towards these substances. The MS is usually operated in Selected Ion Monitoring (SIM) mode measuring only a few ions at a time to achieve enhanced detection limits. To identify the unknown signals the sample was measured again, this time preconcentrating about one litre of air and operating the MS in Scan mode scanning all mass fragments from 15 to 300 atomic mass units (amu). Figure 2 shows the mass spectrum at 8.4 min retention time. The background was subtracted in order to remove peaks originating from air entering the system through small leaks (within the operating parameters recommended by Agilent Technologies) but also from $\mathrm{CO}_{2}$ which elutes at about $4 \mathrm{~min}$ and still showed significantly larger signals on its main fragment (mass-to-charge ratio $m / z$ of 44 ) at 8 min than all other present ions. The result of a search in the NIST mass spectral library (see reference) gave a $97 \%$ probability match with $\mathrm{C}_{2} \mathrm{~F}_{3} \mathrm{Cl}$ (chlorotrifluoroethene). In addition, the second best matching substance had a probability of less than $1 \%$. All important mass fragments were found to be present. The expected relative abundances differed in some cases, which could be caused by the above mentioned interferences from the atmospheric matrix of the plume sample but also by the limited mass resolution of the MS and its reduced sensitivity in the lower $m / z$ range (as compared to NIST spectra).

The substance eluted just after CFC-12 which had a retention time of 8.1 minutes. As the chromatographic system separates primary via boiling points this substance should have a boiling point of about $-20{ }^{\circ} \mathrm{C}$. $\mathrm{C}_{2} \mathrm{~F}_{3} \mathrm{Cl}$ boils at $-28.4^{\circ} \mathrm{C}$. Moreover the pure compound could be obtained from Sigma-Aldrich Corporation (purity: 98\%). It was statically diluted to ppb levels with ultra-pure Nitrogen (from Air Liquide Deutschland GmbH, purity $\geq 99.999 \%$-mol). The subsequent MS-Scan measurements confirmed the identity of the substance by giving a similar mass spectrum at the same retention time which is also shown in Fig. 2. These measurements also confirmed the above mentioned reduced sensitivity of the MS for ions with lower $\mathrm{m} / \mathrm{z}$ ratio.

The mass spectra at 11.1 and 16.2 min retention time gave $99 \%$ probability matches with the NIST library spectra of $\mathrm{CF}_{2} \mathrm{CFCF}_{2} \mathrm{Cl}$ (3-chloropentafluoropropene) and $\mathrm{CF}_{2} \mathrm{CFCF}_{2} \mathrm{CFCl}_{2}$ (4,4-dichlorohexafluoro-1-butene) and the comparisons of spectra are depicted in Figs. 3 and 4. The respective second best matches were below $1 \%$ probability. Both substances boiling points also fitted very well with their retention times. 3-chloropentafluoropropene boils at $8^{\circ} \mathrm{C}$ and eluted $0.45 \mathrm{~min}$ after $\mathrm{CF}_{3} \mathrm{CFCl}_{2}$ (CFC-114a) which boils at $3^{\circ} \mathrm{C}$. For 4,4-dichlorohexafluoro-1-butene boiling points between 67 and $71^{\circ} \mathrm{C}$ have been reported (source: Beilstein Crossfire database) and it coeluted with $\mathrm{CCl}_{4}$ which boils at $77^{\circ} \mathrm{C}$. $\mathrm{CF}_{2} \mathrm{CFCF}_{2} \mathrm{Cl}$ and $\mathrm{CF}_{2} \mathrm{CFCF}_{2} \mathrm{CFCl}_{2}$ could not be obtained as pure compounds by now. But taking into account the agreement between boiling points and mass spectra we consider the identification of the substances as certain. To our knowledge none of the three substances has been re-
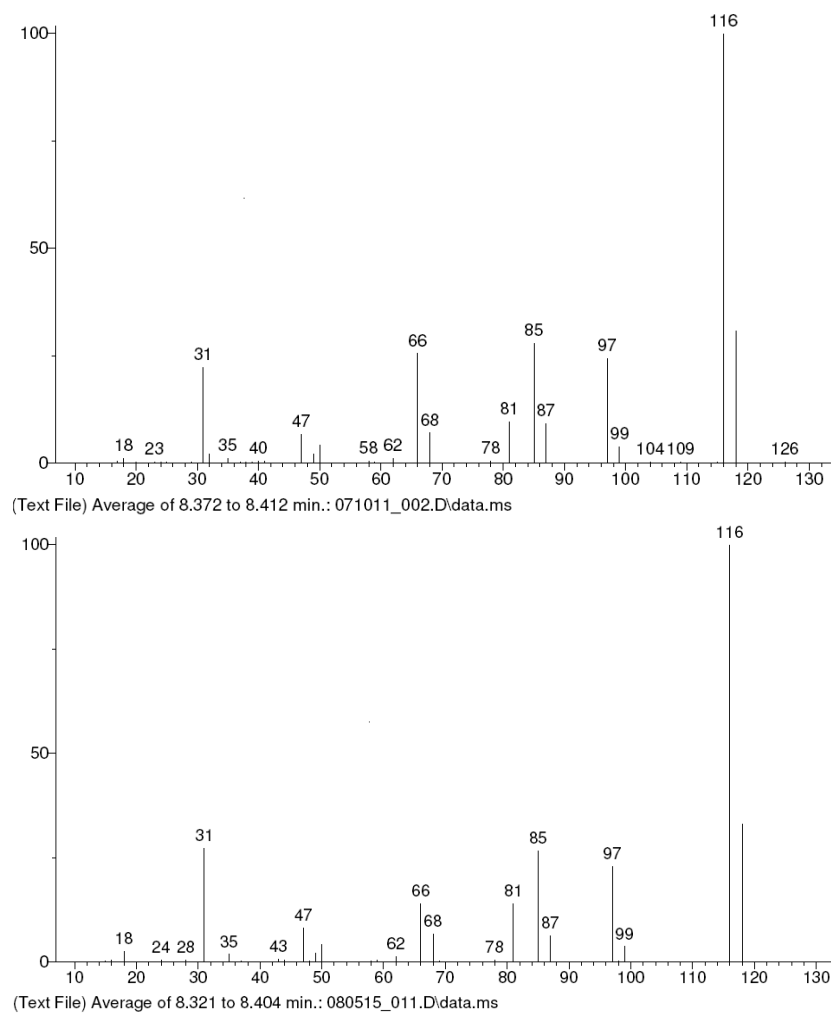

Fig. 2. The upper mass spectrum belongs to a chromatogram of an air sample taken at the Taunus Observatory near Frankfurt. The background spectrum was subtracted in order to remove peaks originating from air entering the system through small leaks and from the decaying signals of $\mathrm{CO}_{2}$ which elutes at about $4 \mathrm{~min}$. Trace gases were concentrated cryogenically from 11 of air and measured by means of GC-MS (EI-Scan mode).The averaged spectrum at about 8.4 min retention time belongs to a substance eluting near $\mathrm{CF}_{2} \mathrm{Cl}_{2}$ (CFC-12) and gave a $97 \%$ probability match with that of chlorotrifluoroethene $\left(\mathrm{C}_{2} \mathrm{~F}_{3} \mathrm{Cl}\right)$ as listed in the NIST mass spectral library. Expected relative abundances for $\mathrm{C}_{2} \mathrm{~F}_{3} \mathrm{Cl}$ were $100 \%$ for mass/charge ratio $(\mathrm{m} / \mathrm{z}) 116,87 \%$ for $m / z 31,38 \%$ for $m / z 66,33 \%$ for $m / z 85,33 \%$ for $m / z 118,31 \%$ for $m / z 97,26 \%$ for $m / z 47,17 \%$ for $m / z 81,13 \%$ for $m / z 68$ and $11 \%$ for $m / z 87$. The lower mass spectrum is that of $\mathrm{C}_{2} \mathrm{~F}_{3} \mathrm{Cl}$ (purity: $98 \%$ ) as obtained from Sigma-Aldrich Corporation which was subsequently diluted to ppb levels with ultra-pure Nitrogen (from Air Liquide Deutschland $\mathrm{GmbH}$, purity $\geq 99.999 \%$-mol) and measured on the same instrument under similar conditions.

ported in the atmosphere before. All other unknown large EDC signals from the plume sample are likely to be caused by halocarbons, too. However, they could not be identified yet due to the fact that comparison with the library gave no exact match. Possible explanations are the coelution of two or more substances or simply missing reference mass spectra.

As no calibration was available for the identified substances in the first place we derived a method for a minimum and maximum concentration estimate. Laube and Engel, 


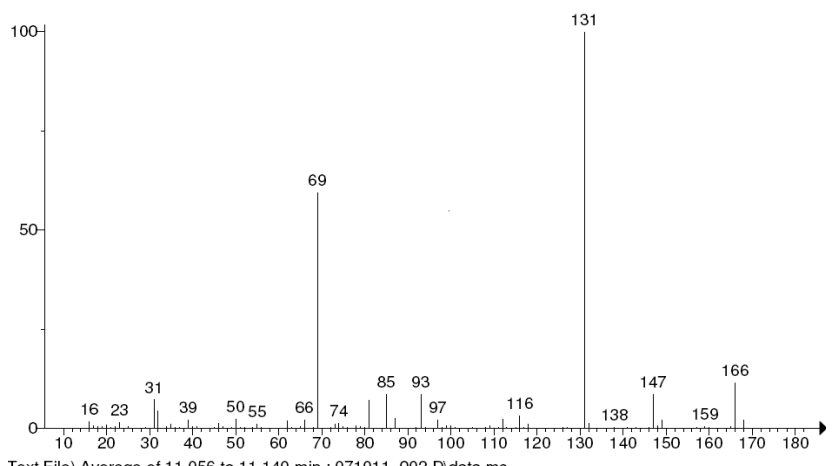

Text File) Average of 11.056 to 11.140 min.: 071011_002.Didata.ms

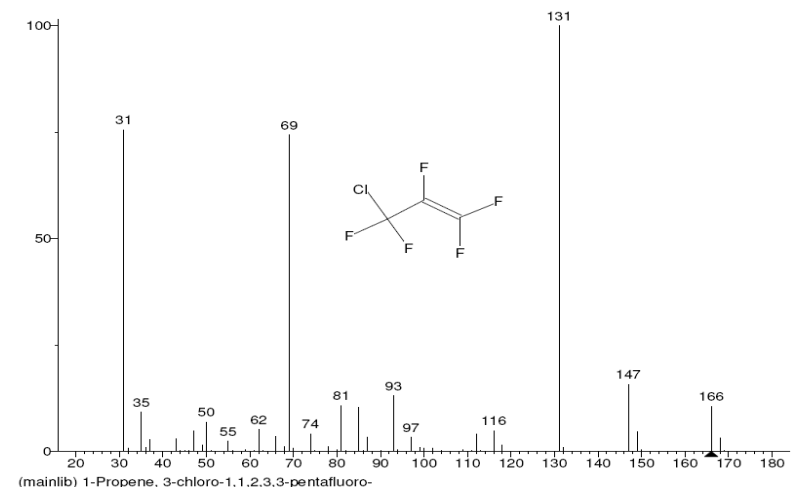

Fig. 3. The same as in Fig. 2 but for $\mathrm{CF}_{2} \mathrm{CFCF}_{2} \mathrm{Cl}$ (3chloropentafluoropropene) at $11.1 \mathrm{~min}$ retention time and the lower spectrum belonging to the NIST library. No pure compound could be obtained in this case but the match probability given by NIST was $99 \%$ and the boiling point $\left(8^{\circ} \mathrm{C}\right)$ fits well because the substance eluted 0.45 minutes after $\mathrm{CF}_{3} \mathrm{CFCl}_{2}(\mathrm{CFC}-114 \mathrm{a})$ which boils at $3^{\circ} \mathrm{C}$.

2008 inferred the sensitivity relative to $\mathrm{CFC}-12$ for six different halocarbons: $\mathrm{CFCl}_{3}(\mathrm{CFC}-11)$ on $\mathrm{m} / z, 103, \mathrm{CF}_{2} \mathrm{ClCFCl}_{2}$ (CFC-113) on $m / z 151, \mathrm{CHF}_{2} \mathrm{Cl}$ (HCFC-22) on $m / z 67$, $\mathrm{CF}_{2} \mathrm{ClBr}$ (Halon-1211) on $m / z 129, \mathrm{CH}_{3} \mathrm{Br}$ on $m / z 94$ and $\mathrm{CHCl}_{3}$ on $m / z$ 83. We improved this method by using an expanded data set from measurements of four different calibration standards on 20 measuring days over a period of two years in order to achieve a better reflection of the MS sensitivity range. Moreover, $\mathrm{C}_{2} \mathrm{Cl}_{4}$ (on $\mathrm{m} / z$ 166) data was included as a seventh substance, because it is chemically very similar to the new substances (fully halogenated and double bond contained). The MS sensitivities to the substances $\left(s_{i}\right)$ were calculated according to Eq. (1).

$s_{i}=h_{i} /\left(a \times \rho_{i}\right)$

$S_{i}=s_{i} / s_{\mathrm{CFC}-12}$

The signal height $h$ of the substance $i$ is divided by the sample amount $a$ and the substance mixing ratio $\rho_{i}$. The relative sensitivity $S_{i}$ is then given by division of $s_{i}$ with $s_{\mathrm{CFC}-12}$ (Eq. 2). Values of $S_{i}$ were between 0.04 and 1.20. To consider the fact that we are using only one mass fragment to

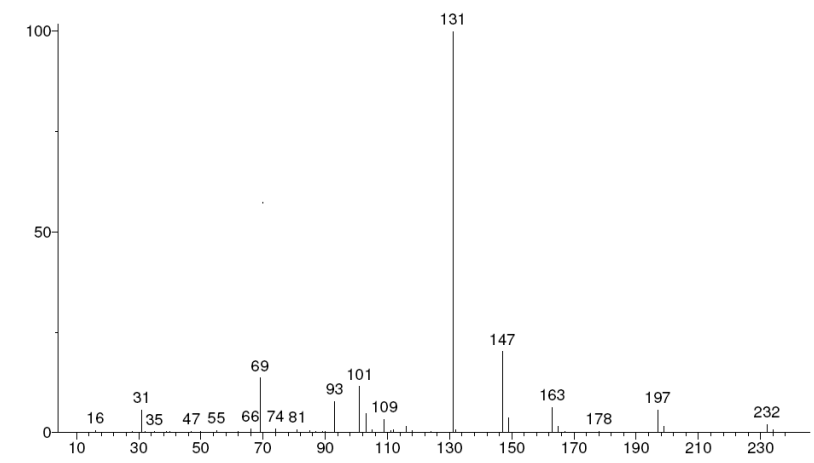

(Text File) Average of 16.189 to 16.228 min.: 071011_002.Dldata.ms

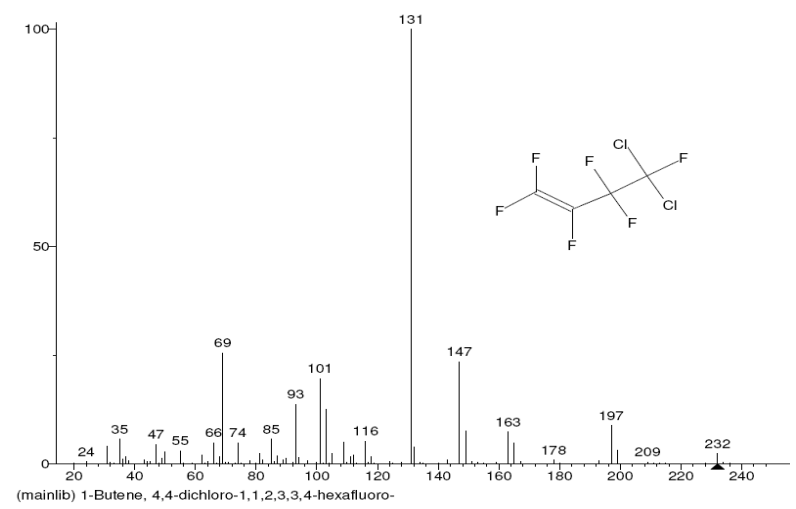

Fig. 4. The same as in Fig. 2 but for $\mathrm{CF}_{2} \mathrm{CFCF}_{2} \mathrm{CFCl}_{2}(4,4-$ dichlorohexafluoro-1-butene) at $16.2 \mathrm{~min}$ retention time and the lower spectrum belonging to the NIST library. No pure compound could be obtained in this case but the match probability given by NIST was $99 \%$ and the reported boiling points $\left(67\right.$ and $\left.71^{\circ} \mathrm{C}\right)$ fit well because the substance coeluted with $\mathrm{CCl}_{4}$ which boils at $77^{\circ} \mathrm{C}$.

quantify a substance we introduced a fragmentation correction factor according to Eq. (3).

$f_{i}=r_{q} / \sum_{j=1}^{j=10} r_{j}$

$C_{i}=S_{i} / f_{i}$

This factor $f_{i}$ is given by $r_{q}$ - the relative abundance of the mass fragment used to quantify the substance - divided by the sum of the relative abundances $r_{j}$ of all major mass fragments. Only fragments with an abundance of more than $10 \%$ relative to the main fragment are used and 10 fragments included at most. The corrected relative sensitivity $C_{i}$ is then given by the relative sensitivity $S_{i}$ divided by the fragmentation factor $f_{i}$ (Eq. 4). Derived values for $C_{i}$ ranged from 0.20 to 3.41. We then calculated minimum and maximum mixing ratios for the new CFCs via the CFC-12 mixing ratio of the sample and derived mixing ratio ranges of $1.1-18.4 \mathrm{ppb}$ for $\mathrm{C}_{2} \mathrm{~F}_{3} \mathrm{Cl}, 0.5-8.8 \mathrm{ppb}$ for $\mathrm{CF}_{2} \mathrm{CFCF}_{2} \mathrm{Cl}$ and $0.6-9.2 \mathrm{ppb}$ for $\mathrm{CF}_{2} \mathrm{CFCF}_{2} \mathrm{CFCl}_{2}$ in the plume observed on 02 October. As mentioned above $\mathrm{C}_{2} \mathrm{~F}_{3} \mathrm{Cl}$ could be obtained later and was diluted to ppb levels (lowest dilution: $4.5 \mathrm{ppb}$ ) in order to 
Table 1. Wind data and mixing ratio estimates of the novel detected CFCs for different air samples taken at the Taunus Observatory. In case of $\mathrm{C}_{2} \mathrm{~F}_{3} \mathrm{Cl}$ the pure compound could be obtained and thus a calibration was possible. The corresponding error bars are an estimate of the sum of all errors from preparation of the calibration standards via static dilution but also include the measurement standard deviations. Please note, that a preconcentration amount error which could originate from the humidity of the samples, was not included. All derived $\mathrm{C}_{2} \mathrm{~F}_{3} \mathrm{Cl}$ mixing ratios did agree with the estimated range from the relative sensitivity method.

\begin{tabular}{lclcccc}
\hline & Wind speed & Wind & Mixing ratio & \multicolumn{3}{c}{ Estimated mixing ratio range [ppt] } \\
Date 2007 & 1h mean [m/s] & Direction & $\mathrm{C}_{2} \mathrm{~F}_{3} \mathrm{Cl}[\mathrm{ppt}]$ & $\mathrm{C}_{2} \mathrm{~F}_{3} \mathrm{Cl}$ & $\mathrm{C}_{3} \mathrm{~F}_{5} \mathrm{Cl}$ & $\mathrm{C}_{4} \mathrm{~F}_{6} \mathrm{Cl}_{2}$ \\
\hline 02 Oct & 1 & $\mathrm{NW}$ & $9800 \pm 1800$ & $1100-18400$ & $530-8800$ & $550-9200$ \\
06 Nov & 6 & $\mathrm{NW}$ & $0.34 \pm 0.09$ & $0.2-3.3$ & $0.2-3.0$ & $0.1-1.2$ \\
$21 \mathrm{Nov}$ & 4 & $\mathrm{~S}$ & $0.41 \pm 0.10$ & $0.2-3.6$ & $0.1-1.4$ & $0.1-1.1$ \\
$05 \mathrm{Dec}$ & 5 & $\mathrm{SW}$ & $0.93 \pm 0.23$ & $0.4-7.1$ & $0.4-6.7$ & $0.1-2.3$ \\
$12 \mathrm{Dec} *$ & $\sim 6$ & $\mathrm{NE}$ & $0.11 \pm 0.03$ & $0.1-1.5$ & $0.2-1.2$ & $0.1-1.0$ \\
$21 \mathrm{Dec}$ & 5 & $\mathrm{SE}$ & $0.23 \pm 0.06$ & $0.1-2.0$ & $0.1-1.7$ & $0.1-1.2$ \\
\hline
\end{tabular}

* sample taken at the Jungfraujoch High Altitude Research Station (Switzerland)

calibrate the measurements. The calculated plume sample mixing ratio was $9.8 \mathrm{ppb}$ which is in agreement with the estimate above.

Trichlorofluoroethene is also known as R-1113 and is toxic. Cook and Pierce, 1973 reported an $\mathrm{LC}_{50}$ (i.e. the concentration which kills $50 \%$ of a sample population) of 1000 ppm and its ERPG-3 (i.e. the maximum airborne concentration below which individuals could be exposed for up to $1 \mathrm{~h}$ without life-threatening health effects) is $300 \mathrm{ppm}$ (source: http://cameochemicals.noaa.gov). Like most fluoroalkenes 3-chloropentafluoropropene and an isomer of dichlorohexafluorobutene are also reported to be highly toxic (Thun and Kimbrough, 1981; Clayton, 1977). Considering the fact that $\mathrm{C}_{2} \mathrm{~F}_{3} \mathrm{Cl}$ carries a double bound and is highly flammable its atmospheric lifetime is expected to be very short. Thus we suggest the plume we observed originated from a local source which is supported by the low wind speed $(\sim 1 \mathrm{~m} / \mathrm{s})$ on the sampling day. However, concentrations close to the source could have been much higher - especially if that source was located indoors. We observed other large unknown signals which are likely to be chlorofluoroalkenes due the occurrence of characteristic fragments like $m / z 147$ and 149 or $m / z 197$ and 199. Both pairs showed an abundance ratio of $3: 1$ which is - in combination with the high ECD sensitivity - an indication towards the $\mathrm{C}_{2} \mathrm{~F}_{4} \mathrm{Cl}^{+}$and the $\mathrm{C}_{3} \mathrm{~F}_{6} \mathrm{Cl}^{+}$fragments. Thus it can not be ruled out that the sum of the chlorofluoroalkenes could have reached concentrations which affect human health. On the Kleiner Feldberg six days backward trajectories on different pressure levels are calculated regularly with the DWD-GME model operated by the German Weather Service (DWD), Offenbach, Germany. They showed that air masses had been advected from the southwest sector with rather low wind speed probably originating from the densely populated Rhein-Main area.

We took six more air samples at the Taunus observatory and one at the High Altitude Research Station Jungfraujoch (Switzerland) and measured them by running the MS in SIM mode monitoring $\mathrm{C}_{2} \mathrm{~F}_{3} \mathrm{Cl}$ on $\mathrm{m} / z, 116$ for quantification and 118 for confirmation, $\mathrm{CF}_{2} \mathrm{CFCF}_{2} \mathrm{Cl}$ on $\mathrm{m} / z 131$ (q) and 166 (c) and $\mathrm{CF}_{2} \mathrm{CFCF}_{2} \mathrm{CFCl}_{2}$ on $m / z 147$ (q) and 131 (c). The substances were present all the time except for the sample taken directly (but still using the sampling line) at the exhaust of the observatories air conditioning system which was free of short-lived CFCs. An indoor sample taken at our institute contained no $\mathrm{C}_{2} \mathrm{~F}_{3} \mathrm{Cl}$ and no $\mathrm{CF}_{2} \mathrm{CFCF}_{2} \mathrm{Cl}$ but a very small amount of $\mathrm{CF}_{2} \mathrm{CFCF}_{2} \mathrm{CFCl}_{2}$. The estimated mixing ratios for the six outdoor samples were in the lower and sub-ppt range and can be found in Table 1. The corresponding MS signals were reintegrated compared to Laube and Engel, 2008 by using an improved version of the integration software.

The seven samples were also used to evaluate the relative sensitivity method. Mixing ratio ranges were predicted for nine calibrated compounds. The calibration-derived mixing ratios of $\mathrm{CFC}-11, \mathrm{HCFC}-22, \mathrm{H}-1211$ and $\mathrm{CHCl}_{3}$ on the above-mentioned ions and in addition of $\mathrm{CCF}_{2} \mathrm{ClCF}_{2} \mathrm{Cl}$ (CFC-114) on $m / z 135, \mathrm{C}_{2} \mathrm{~F}_{5} \mathrm{Cl}$ (CFC-115) on $m / z$ 85, $\mathrm{CCl}_{4}$ on $m / z, 117$ and $\mathrm{CH}_{3} \mathrm{Cl}$ on $\mathrm{m} / z 50$ were found to agree with the estimated ranges from the relative sensitivity method for all seven air samples. Not evaluated were $\mathrm{H} 1211, \mathrm{CHCl}_{3}$, CFC-114 and CFC-115 in the plume sample because their abundances were below detection limits in scan mode and also six of the substances in the sample from November 6, because only CFC-114 and CFC-115 were measured. In case of $\mathrm{C}_{2} \mathrm{~F}_{3} \mathrm{Cl}$ a subsequent calibration was carried out and all calculated mixing ratios agreed with the estimated ranges, which is shown in Table 1. Thus, the improved relative sensitivity method can be considered as a useful tool to provide first indications of halocarbon mixing ratios in air samples.

Due to the few data no correlation with wind speed or wind direction could be observed. The three short-lived CFCs were all present in the sample collected at the Jungfraujoch though we can not rule out that they were emitted from the station itself. We also checked several of our air standards for the substances. All of them contained low amounts of 
the CFCs and two even showed contaminations with $\mathrm{C}_{2} \mathrm{~F}_{3} \mathrm{Cl}$ probably originating from the pressure regulator or the cylinder valve. The properties of $\mathrm{C}_{2} \mathrm{~F}_{3} \mathrm{Cl}$ are very similar to those of CFC-12 (boiling points and several main fragments such as $m / z 31,35,50,66,85$ and 87$)$. Thus, coelution is very likely to occur in other chromatographic systems. Contaminations could cause falsified calculations of CFC-12 mixing ratios if an ECD or MS is used for detection.

We believe the observed substances are very likely to be connected with chlorofluoro(co)polymers - particularly polychlorotrifluoroethene (PCTFE, tradenames are Kel-F, Neoflon or Aclar). PCTFE was first commercialized in 1934 by Hoechst (Utracki, 1995) and is still widely used in equipment manufacturing because of its excellent thermoplastic properties, chemical resistance and good impermeability to gases and vapours (Abusleme and Manzoni, 2004). The main precursor for PCTFE production is chlorotrifluoroethene. Birnbaum et al., 1968 studied the toxicity of the pyrolysis of PCTFE and found it to increase rapidly with rising temperature. The thermal degradation of PCTFE and copolymers of it were studied by Zulfiqar et al., 1994 who found the monomer (i.e. chlorotrifluoroethene) to be the major product and amongst others chloropentafluoropropene in traces. Long et al., 1984 carried out infrared multiphoton dissociation experiments and found the toxic substances $\mathrm{COF}_{2}$, $\mathrm{COFCl}$, and $\mathrm{CF}_{2} \mathrm{ClCOF}$ to be the exclusive products in the presence of oxygen. We checked the MS scan chromatogram of the plume sample but could not detect any of these product gases. The microbial biodegradation of $\mathrm{CF}_{2} \mathrm{ClCFCl}_{2}$ (CFC-113) and $\mathrm{CHClFCClF}_{2}$ (HCFC-123a) are also possible sources of chlorotrifluoroethene. A detailed overview on that topic was given by Field and Sierra-Alvarez in 2004. But as 3-chloropentafluoropropene and dichlorohexafluorobutenes are also used for the production of copolymers (e.g. Robb et al., 1962; Lo et al., 1959) our suggestion is, that the observed high abundances were generated by the thermal degradation of a fluoropolymer blend.

\section{Conclusions}

We have shown that chlorotrifluoroethene, 3chloropentafluoropropene and 4,4-dichlorohexafluoro1-butene are present in the atmosphere. The substances were identified through comparison of their mass spectra with the NIST library and their retention times were in agreement with the expectation based on the boiling point. We have also observed other substances which are suspected to be chlorofluoroalkenes but were not able to identify them up to now. The identified CFCs showed high mixing ratios in the lower ppb range in a plume but the substances were also observed to be present in background ambient air with mixing ratios in the lower and sub-ppt range. These CFCs are not listed in the Scientific Assessment of Ozone Depletion 2006 of the World Meteorological Organisation/United Nations
Environment Programme (WMO/UNEP) (see Tables 1-4 of Clerbaux and Cunnold, 2007) or the Montreal Protocol and its subsequent amendments. The substances are most probable of anthropogenic origin. But although their contribution to ozone depletion can be expected to be rather small it is important to find out more about their sinks and sources and their ability to reach the stratosphere. Furthermore all known CFCs are strong greenhouse gases. Thus we suggest to establish continuous measurements of these substances and to investigate their atmospheric lifetimes in order to assess their possible influence on the global background atmosphere. Considering human health it is also important to find out if plumes containing high amounts of the CFCs can occur again - outdoor as well as indoor.

Acknowledgements. We would like to thank A. Werner for taking air samples, the University of Frankfurt for supporting the operation of the Taunus Observatory, the Swiss Federal Laboratories for Materials, Testing and Research (EMPA, especially M. Steinbacher), the High Altitude Research Station Jungfraujoch, MeteoSchweiz and the Hessisches Landesamt für Umwelt und Geologie for providing wind data, the German Weather Service for providing backward trajectories and the DFG project CAWSES (EN 367/4) as well as the EU project SCOUT-O3 (GOCE-CT-2003-505390) for funding. Moreover we express our gratitude to the anonymous reviewers for their essential contribution to the manuscript.

Edited by: J. Kaiser

\section{References}

Abusleme, J. A. and Manzoni, C.: Synthesis of thermoplastic perhalogenated (co)polymers of chlorotrifluoroethylene, European Patent, EP1067146, 2004.

Birnbaum, H. A., Scheel, L. D., and Coleman, W. E.: The toxicology of the pyrolysis products of polychlorotrifluoroethylene, Am. Ind. Hyg. Assoc. J., 29, 61-65, 1968.

Clayton, J. W.: Toxicology of the fluoroalkenes: review and research needs, Environ. Health Persp., 21, 255-267, 1977.

Clerbaux, C., Cunnold, D. M., Anderson, J., et al.: Halogenated Long-lived Compounds, Scientific assessment of ozone depletion: 2006, Global Ozone Research and Monitoring Project, Report No. 50, Chapter 1, World Meteorological Organization, Geneva, Switzerland, 2007.

Cook, E. W. and Pierce, J. S.: Toxicology of fluoro-olefins, Nature, 242, 337-338, 1973.

Field, J. A. and Sierra-Alvarez, R.: Biodegradability of chlorinated solvents and related chlorinated aliphatic compounds, Rev. Environ. Sci. Biotechnol., 3, 185-254, 2004.

Laube, J. C. and Engel, A.: First atmospheric observations of three chlorofluorocarbons, Atmos. Chem. Phys. Discuss., 8, 66836695, 2008,

http://www.atmos-chem-phys-discuss.net/8/6683/2008/.

Law, K. S., Sturges, W. T., Blake, D. R., et al.: Halogenated very short-lived substances, Scientific assessment of ozone depletion: 2006, Global Ozone Research and Monitoring Project, World Meteorological Organization, Geneva, Switzerland, Report No. 50, Chapter 2, 2007. 
Lo, E. S. and Elizabeth, N. J.: Copolymers of dichlorohexafluorobutene, US-Patent 2914514, 1959.

Long, G. R., Prentice, L. D., and Bialkowski, S. E.: Chemical reactions following the IRMPD of $\mathrm{C}_{2} \mathrm{~F}_{3} \mathrm{Cl}$, Appl. Phys. B-Photo, 34, 97-106, 1984.

National Institute of Standards and Technology: Mass Spectral Search Program for the NIST/EPA/NIH Mass Spectral Library, Version $2.0 \mathrm{~d}$, National Institute of Standards and Technology, Gaithersburg, MD, USA, 2005.

Reimann, S., Vollmer, M. K., Folini, D., Steinbacher, M., Hill, M., Buchmann, B., Zander, R., and Mahieu, E.: Observations of Long-Lived Anthropogenic Halocarbons at the High-Alpine site of Jungfraujoch (Switzerland) for Assessment of Trends and European Sources, Sci. Total Environ., 391(2-3), 224-231, 2008.
Robb, L. E. and Westfield, N. J.: Polyblends of a thermoplastic tetra-fluoroethylene polymer, US-Patent 3019206, 1962.

Solomon, S.: Stratospheric ozone depletion: a review of concepts and history, Rev. Geophys., 37, 275-316, 1999.

Thun, M. and Kimbrough, R. D.: Fatal chemical pneumonia from 1,1,2,3,3-pentafluoro-3-chloropropene in an unmarked gas tank, Clin. Toxicol., 18, 481-487, 1981.

Utracki, L. A.: History of commercial polymer alloys and blends (from a perspective of the patent literature), Polym. Eng. Sci, 35, 2-17, 1995.

Zulfiqar, S., Zulfiqar, M., Rizvi, M., Munir, A., and McNeill, I. C.: Study of the thermal degradation of polychlorotrifluoroethylene, poly(vinylidene fluoride) and copolymers of chlorotrifluoroethylene and vinylidene fluoride, Polym. Degrad. Stabil., 43, 423430, 1994. 